\title{
Study of the Adhesive Coefficient Effect on the Hydraulic Losses and Cavitation
}

\author{
František Pochylý1 ${ }^{1}$ Simona Fialová ${ }^{1}$, Milada Kozubková², Lukáš Zavadil ${ }^{2}$ \\ ${ }^{1}$ Faculty of Mechanical Engineering, Brno University of Technology \\ Technická 2896/2, Brno, 616 69, Czech Republic, pochyly@fme.vutbr.cz, fialova@fme.vutbr.cz \\ ${ }^{2}$ Faculty of Mechanical Engineering, Technical University of Ostrava \\ 17. listopadu 15/2172, Ostrava - Poruba, 708 33, Czech Republic, milada.kozubkova@vsb.cz
}

\begin{abstract}
The article is focused in three areas. In the first part there are analyzed the adhesion forces at the liquid and solid surface interface. There are shown the measured values of surface energy for different types of surfaces. The value of surface energy is decisive for determining the extent of the surface wettability by the liquid. The second part points to the possible negative effects of partly wettable surfaces, showing susceptibility to cavitation. The third section describes the practical aspects of surface wettability by the liquid. Under the new boundary conditions bases, expressing the effect of adhesion forces, there are determined the centrifugal pump characteristics.
\end{abstract}

Keywords: cavitation, wettability, adhesion force, impeller pump

\section{Introduction}

Beginning of the 21 st century is characterized by save efforts of electricity produced by burning fossil fuels. Preferences are given to wind and solar farms build up, whose output can be regulated only by significant additional costs.

Significant energy savings can be achieved much more elegant way, by reducing the hydraulic losses, using the latest scientific knowledge related to nanotechnology applications.

Generally it is argued that one quarter of all produced energy is consumed for liquids pumping. Increasing the efficiency of hydraulic systems for at least two percent would mean enormous savings in electricity. And this can be achieved by reducing the hydraulic losses.

The biggest part of the hydraulic losses arises of the fluid flow near the surface and depends on fluid velocity gradient in the normal direction to the surface. Velocity gradient is subject to the condition of liquid adhesion to the surface, which reflects the fact that the flowing liquid to a stationary surface has zero speed. See the shape of the velocity profile, as shown in Fig.1).

Sticking condition is fulfilled for the majority of known compounds (metals - water, water - glass, metals - oil, most plastics water, etc.).

Reducing adhesion to the surface of the flowing liquid can be achieved by reducing the gradient of liquid velocity on liquid slip on a rigid surface, thereby reducing hydraulic losses. See Fig. 1b).

The aim of this paper is to show phenomena that can occur; we can - if physical methods to reduce the adhesion strength in the surface layer of liquid to solid liquid interface.

a)

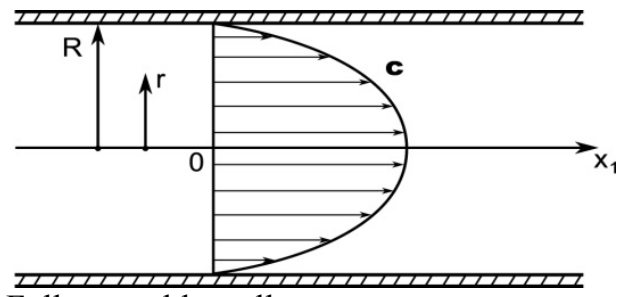

a) Fully wettable wall

b) Partially wettable wall

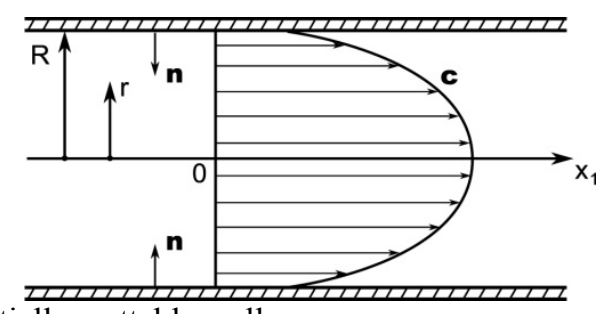

Fig. 1 Velocity profiles of the liquid

Accepted for publication December 14, 2010: Paper number O10044S

Corresponding author: Simona Fialova, Assistant Professor, fialova@fme.vutbr.cz

This manuscript was presented at the $25^{\text {th }}$ IAHR Symposium on Hydraulic Machinery and Systems, September 20-24, 2010, Politechnica University of Timisoara, Romania . 


\section{Surface energy [2]}

Liquids in contact with the insoluble solid surface rely on three inter phase energies: solid surface energy $\gamma_{\mathrm{sg}}$, interfacial energy solid - liquid $\gamma_{\mathrm{sl}}$ and liquid surface energy $\gamma_{\mathrm{g}}$; their relative values affect the resulting interface surface energy, which we deem as a factor (ratio) of surface wettability by the liquid. The size of the surface energy can be determined by contact angle $\theta$ of liquid droplets on the selected species surface. See Fig 2.
(a) $\theta=0^{\circ}$
(b) $0^{\circ}<\theta<90^{\circ}$

(c) $90^{\circ}<\theta<180^{\circ}$

(d) $\theta=180^{\circ}$

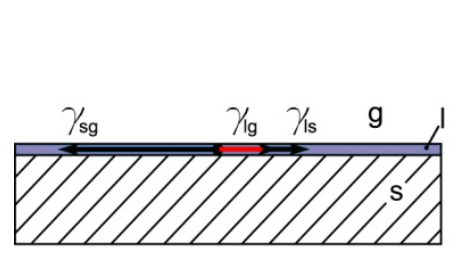

$$
\gamma_{\mathrm{sg}}=\gamma_{\mathrm{g}}+\gamma_{\mathrm{s}}
$$

absolute wetting
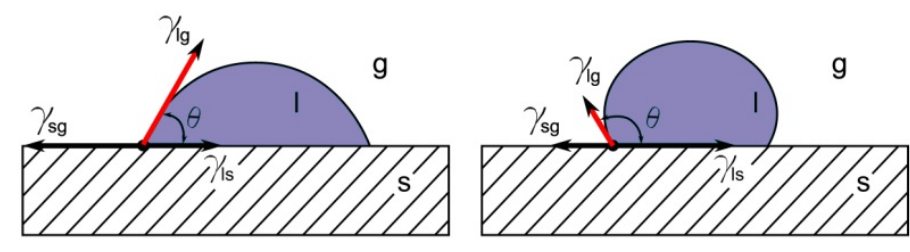

$$
\gamma_{\mathrm{sg}}>\gamma_{\mathrm{ss}}-\gamma_{\mathrm{sg}}<\gamma_{\mathrm{ss}}+\gamma_{\mathrm{gg}}-\gamma_{\mathrm{sg}}<\gamma_{\mathrm{ss}}
$$

good wetting bad wetting

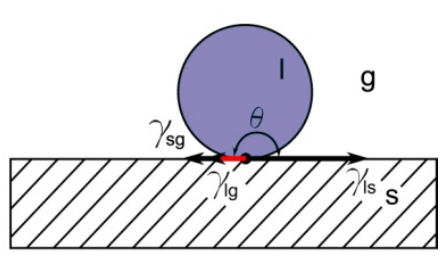

$$
\gamma_{\mathrm{sg}}=\gamma_{\mathrm{ss}}-\gamma_{\mathrm{g}}
$$

absolute nonwetting

Fig. 2 Dependence of the surface wettability on the contact angle $\theta$

On the surface energy basis or the contact angle measuring is advised the degree of liquid wettability to the surface. The situation is illustrated in Fig 2.

Based on this methodology, were performed measurements of surface energy on the SEE System device equipped with a camera to capture the shape of liquid droplets on a selected surface. Based on the contact angle of surface energy are evaluated for various combinations of liquids and surfaces. TC designation indicates the different qualities of Teflon layer deposited on steel plate. The results are tabulated below.

The results indicate that the glass surface, out of tabulated surfaces types, is one of low contact angle and high surface energy, it means is hydrophilic to water. Glass is completely wetted with water and sticking condition applies here. By contrast, for example, Teflon coating (Green TC 4111) is to water non wettable, which corresponds to the high contact angle and low surface energy.

The proof is obvious from another experiment; illustrated in Fig 5. Here is visualized the water flow down a slippery slope. On Fig. 3 a) due to sticking on the surface of a glass of water run-off occurs in the intact layer, which creates a continuous film here. Fig. 3 b) shows the water flow down the slippery slope, under the same conditions as in the case of 5a). Because the green Teflon surface has very low surface energy, the adhesion forces affect water flow only marginally. So in the movement of water the are applied especially surface tension forces and water run-off has character of braids, which perform unsteady motion on an inclined plane. See Fig 5b).

\begin{tabular}{|l|c|c|c|c|}
\hline \multicolumn{1}{|c|}{ Specimen } & \multicolumn{3}{|c|}{ Contact angle $\left(^{\circ}\right)$} & Surface energy \\
\hline & water & ethylene glycol & a-bromnaftalen & $\left(\mathrm{MJ} / \mathrm{m}^{2}\right)$ \\
\hline Black metallic (TC 3072) & $107.1 \pm 4.4$ & $90.9 \pm 2.9$ & $68.1 \pm 4.9$ & 24 \\
\hline Black (TC 1191) & $94.1 \pm 1.3$ & $65.3 \pm 5.6$ & $44.8 \pm 5.8$ & 34 \\
\hline White (aircraft plate) & $78.7 \pm 3.2$ & $49.5 \pm 5.5$ & $19.9 \pm 2.9$ & 43 \\
\hline Glass & $34.6 \pm 4.6$ & $30.7 \pm 3.8$ & $26.8 \pm 5.6$ & 50 \\
\hline Green (TC 4111) & $109.2 \pm 6.2$ & $84.9 \pm 5.1$ & $64.2 \pm 11.9$ & 23 \\
\hline
\end{tabular}

Contact angle and surface energy measurements before coating

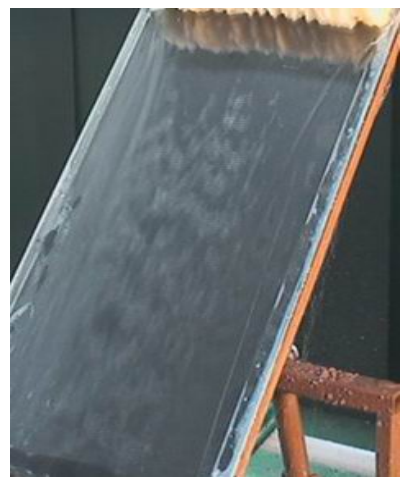

a)

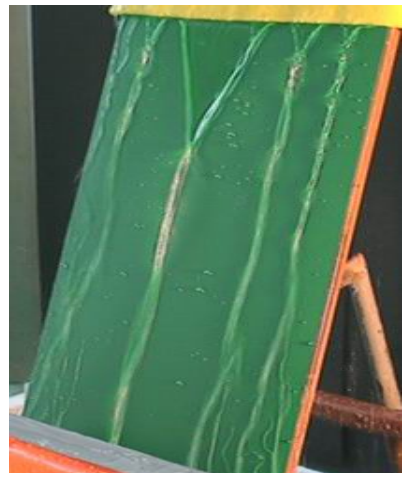

b)

Fig. 3 Water drop down on an inclined plane 


\section{Boundary condition - adhesive forces effect [3]}

For the Navier-Stokes equations and continuity equation solving there must be specified boundary conditions on the interface of the surface and the liquid. In most of the previously solved problems there are expected a liquid that perfectly wetted the surface, so it is possible to explain the liquid sticking condition to the immobile surface by zero velocity vector c, so:

$$
\mathbf{c}=\mathbf{0}
$$

From Fig.1) it is clear that this condition is essential for the flow profile creating.

In the introduction we mentioned the influence of the flow profile on the hydraulic losses.

The influence of high velocity gradients at the wall have a significant effect on the size of the convective acceleration, which affects the stability of laminar flow and are a source of turbulence. Will - when the surface is hydrophilic, means non wettable to the fluid, it changes significantly the boundary condition (1), whereas that for hydrophilic surfaces the liquid will slip against surface. See Fig.1b).

This will change as the hydraulic losses as the size of the convective acceleration and turbulence conditions. Similarity number, characterizing the transition between laminar and turbulent flow, will depend on surface energy.

Boundary condition at the body-fluid interface was discussed in the Navier [3] nearly 200 years ago and he also designed its basic shape, which expresses the slip of liquid on the surface

$$
c=\beta \cdot \frac{d c}{d r}
$$

He assumed that the velocity on the surface of the body is proportional to the shear stress on the surface; where $\beta$ is the slip coefficient. If $\beta=0$ than equation (2) will reflect the sticking condition (1). When coefficient $\beta$ will have the final size than the liquid slippage happens on the wall.

Expression (2) can be used to solve planar flow. Not, however, for the generally curved surface. For this purpose it is necessary to derive a new condition, which must also include a further requirement for the impermeable border, which can be expressed in the form:

$$
c \cdot n=0
$$

Assuming that the adhesive shear stress vector $\sigma_{A}$ lays in the plane intended by the outer normal vector $\mathbf{n}$ to the surface and the velocity vector c. Based on this concept can be defined (2) in assume that the vector of adhesive shear stress on the partially wettable surface is proportional to the speed of the liquid.

Under this assumption, the adhesive shear stress vector $\sigma_{A}$ is true

$\sigma_{A}=(\sigma \times \mathbf{n}) \times \mathbf{n}=-k \mathbf{c}$, as components of this relationship takes the following form

$$
\sigma_{j} n_{j} n_{i}-\sigma_{i}=-k c_{i}
$$

If we substitute the irreversible stress vector like:

$$
\begin{gathered}
\sigma_{i}=\prod_{i j} n_{j}, \quad \text { we can achieve after substitution into (5) the following: } \\
\sigma_{A i}=\prod_{j k} n_{k} n_{j} n_{i}-\Pi_{i j} n_{j}=-k c_{i}
\end{gathered}
$$

Relation (6) defines the boundary condition for liquid slipping on the non wettable, generally curved surface, including the impermeability boundary condition (3).

Adhesive coefficient reflects the degree of adhesion of liquid to the surface and is related to surface energy. It is not yet enough information about whether a given type of surface is constant, independent of the speed $\mathbf{c}$ and the surface roughness. 


\section{Example of fluid slippage flow}

Laminar flow in a tube with circular cross section

On Fig.1), b) is indicated basic signs, and below basic physical constants and dimensions.

\begin{tabular}{|c|c|c|c|}
\hline \multicolumn{4}{|l|}{ Pipeline parameters } \\
\hline Pipeline diameter & $d=$ & 0,015 & $\mathrm{M}$ \\
\hline Pipeline length & $l=$ & 1 & $\mathrm{M}$ \\
\hline Average speed of liquid & $v=$ & 0,1 & $\mathrm{~m} \cdot \mathrm{s}^{-1}$ \\
\hline $\begin{array}{l}\text { Number of computer network } \\
\text { cells }\end{array}$ & $n=$ & 771012 & 1 \\
\hline \multicolumn{4}{|l|}{ Liquid } \\
\hline Liquid density & $\rho=$ & 998,2 & $\mathrm{~kg} \cdot \mathrm{m}^{-3}$ \\
\hline Dynamical viscosity & $\eta=$ & 0,001003 & Pa.s \\
\hline Kinematical viscosity & $v=$ & $1,00481 \mathrm{E}-06$ & $\mathrm{~m}^{2} \cdot \mathrm{s}^{-1}$ \\
\hline
\end{tabular}

Boundary condition (6) on the tube wall is by adjusting in the form:

$$
-\left.\frac{\partial c}{\partial r}\right|_{r=R}=\frac{k}{\eta} c
$$

Solution of the Navier-Stokes equations and continuity equation gives the following velocity profile shape, depending on the adhesive coefficient $k$ :

$$
c=\frac{p_{1}-p_{2}}{2 l}\left(\frac{R^{2}}{2 \eta}+\frac{R}{k}-\frac{r^{2}}{2 \eta}\right)
$$

Flow is given by expression:

$$
Q=\frac{p_{1}-p_{2}}{8 \eta l} \pi R^{4}\left(1+\frac{4 \eta}{k R}\right)
$$

Velocity profile shapes depending on adhesive coefficient are shown in Fig (4).

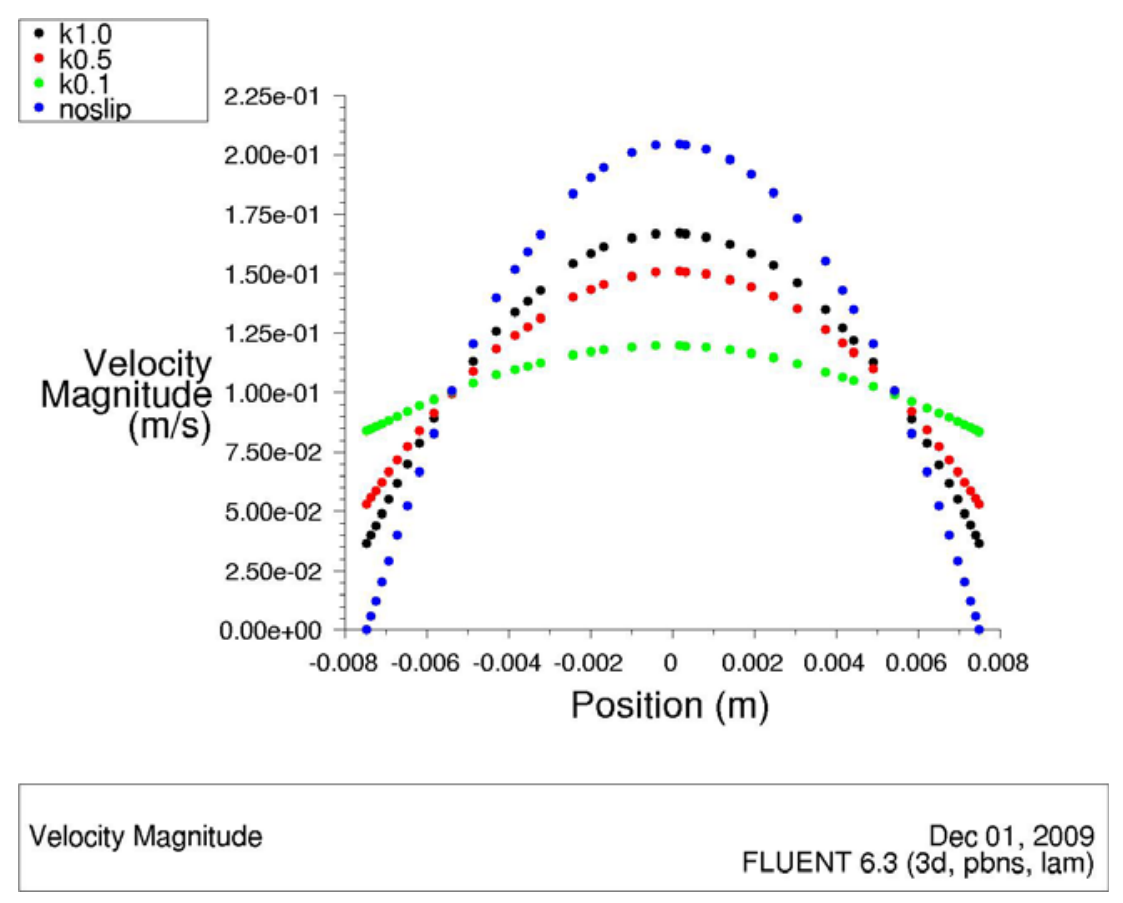

Fig. 4 Liquid velocity profile in adhesive coefficient dependence 
From the figure is evident the significant influence of adhesive coefficient $k$ to shape the flow profile. From here we can also expect a significant impact on the hydraulic losses and wall features that will fundamentally affect the turbulence models.

The following figure (Fig. 5) shows a loss coefficient based on adhesive coefficient.

The opinion is clear that the essential from the loss point of view will have a surface with the adhesive coefficient smaller than $2 \mathrm{Pas} / \mathrm{m}$.

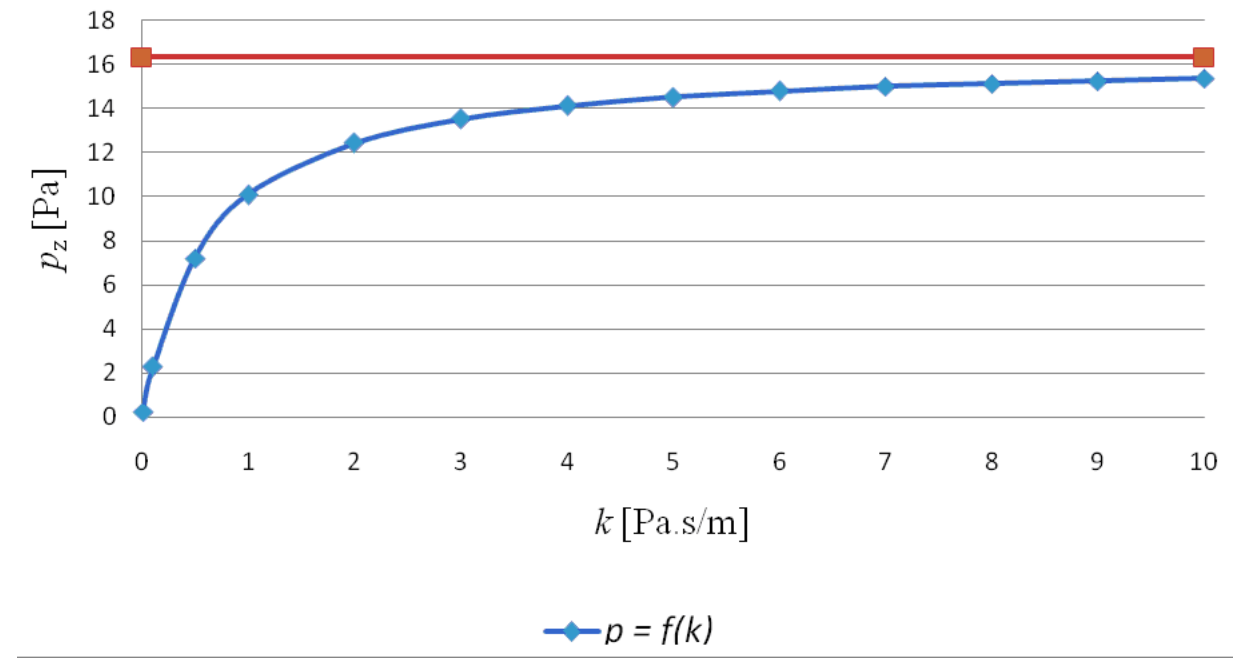

Fig. 5 Pressure losses in adhesive coefficient dependence

\section{Cavitation}

The liquid slipping along the surface will have a significant impact not only on the wall functions, but will also affect the conditions of cavitation creation and suction capacity of hydraulic machines. For this purpose, a numerical experiment was conducted on which basis was possible to evaluate the adhesive coefficient effect for the cavitation cloud development. Situation is shown in Fig.6, where the pressure field is specified in the special cavitation tube. The view shows obviously the expected result of the cavitation area increase in non wettable surface, at $\mathrm{k}=1 \mathrm{Pas} / \mathrm{m}$.

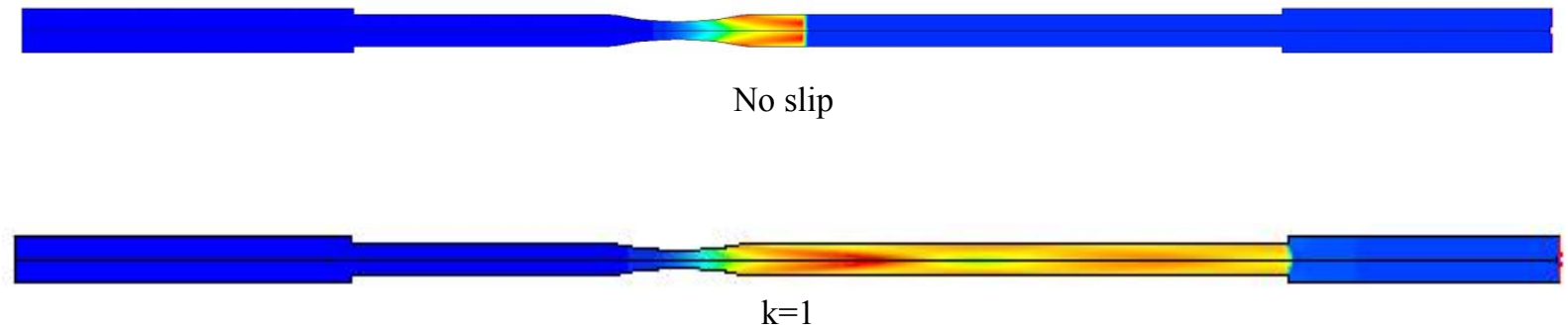

Fig. 6 Cavitation zone for no slip $\mathrm{k}=0$ and partially slip surface $\mathrm{k}=1$ (calculation)

We note that cavitation cloud computational modeling by software FLUENT in the cavitation tube with perfectly wettable surface was confirmed by experiment. Cavitation cloud found in the experiment was shown in Fig.7 and compared with calculation. 

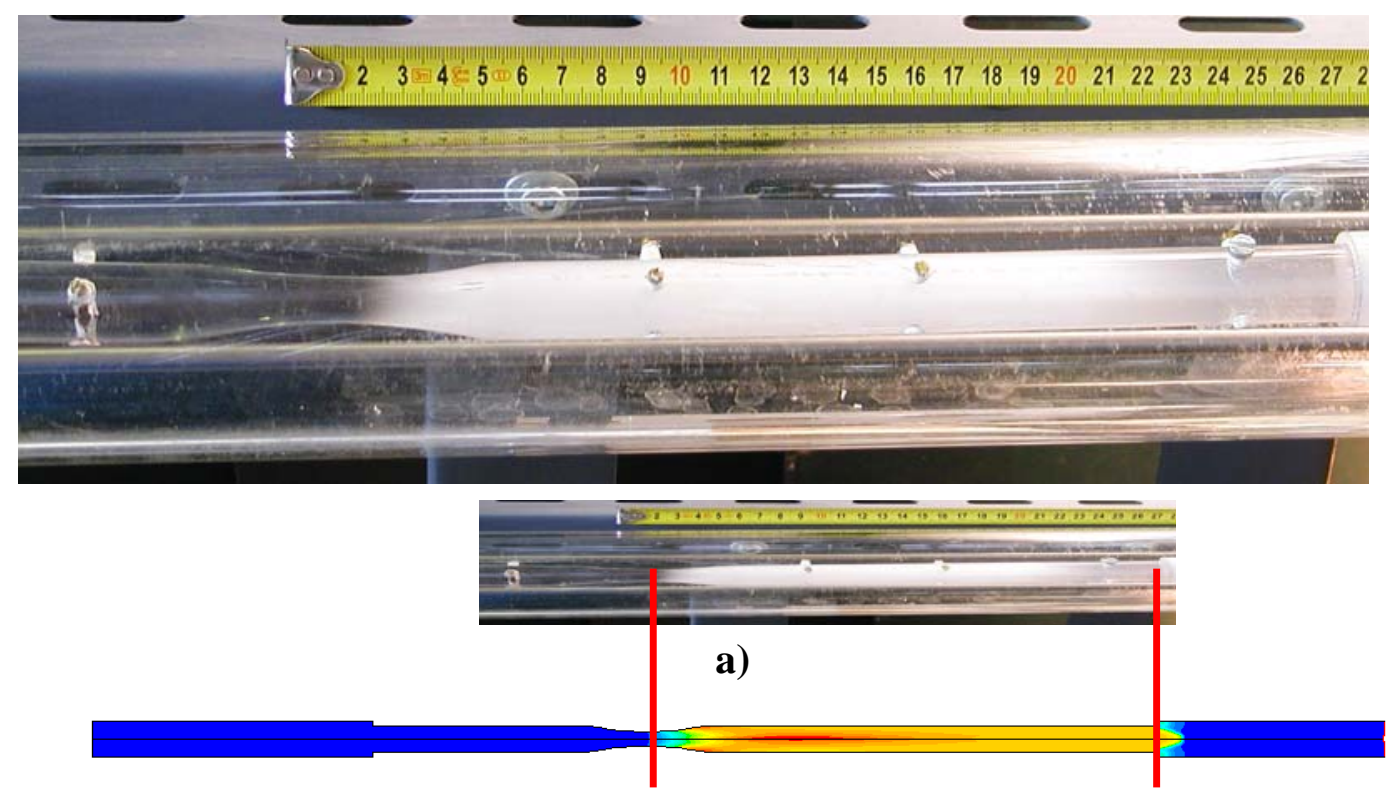

b)

Fig. 7 Cavitation cloud in a tube with a perfect wettable surface a) - experiment b)-calculation

More obvious is the adhesive coefficient effect on the cavitation creation visible from fig. 8 .
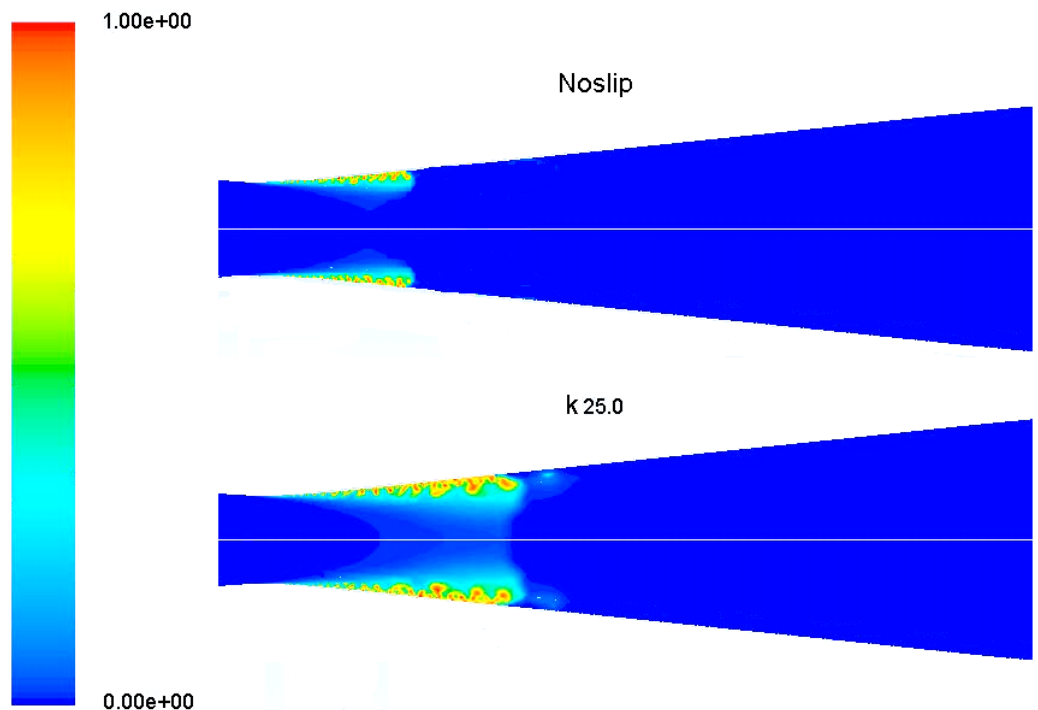

$0.00 e+00$

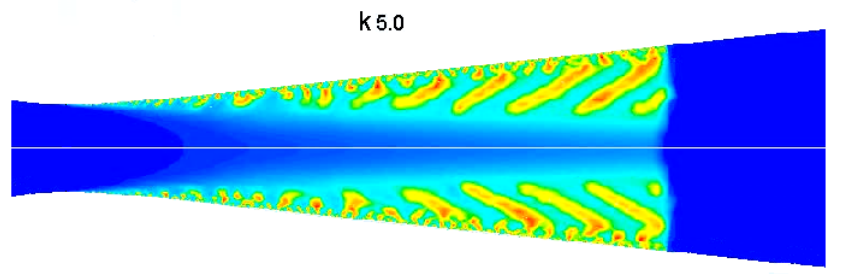

Contours of Volume fraction (Vapour)

ANSYS FLUENT 12.1 (axi, dp, pbns, mixture, rngke, transient)

Fig. 8 Contours of volume fraction (Vapour)

The above mentioned analysis shows that wettability will significantly affect the properties of hydraulic components. Positive is reduction of hydraulic losses. Adverse effects of wettability, however, can result in greater vulnerability to cavitation of hydraulic components.

In the following section we apply the previous expectation of the centrifugal pump characteristics changing. The object of interest will be the adhesive coefficient influence. 


\section{Characteristics of a centrifugal pump with partial wettable surface}

Fig.9 shows the characteristics of centrifugal pumps with low specific speed. It is assumed that the properties of the low wettable surface have only spiral body interior. On the impeller is fulfilled a condition of full wettability. The following results shown in Figures 9, 10, 11 were realized by computational modeling in FLUENT environment.

It is clear that the adhesive coefficient has significant influence on the pump head. The adhesive coefficient reduction may have a negative impact on the stability of (H, Q) characteristic. See Fig. 9.

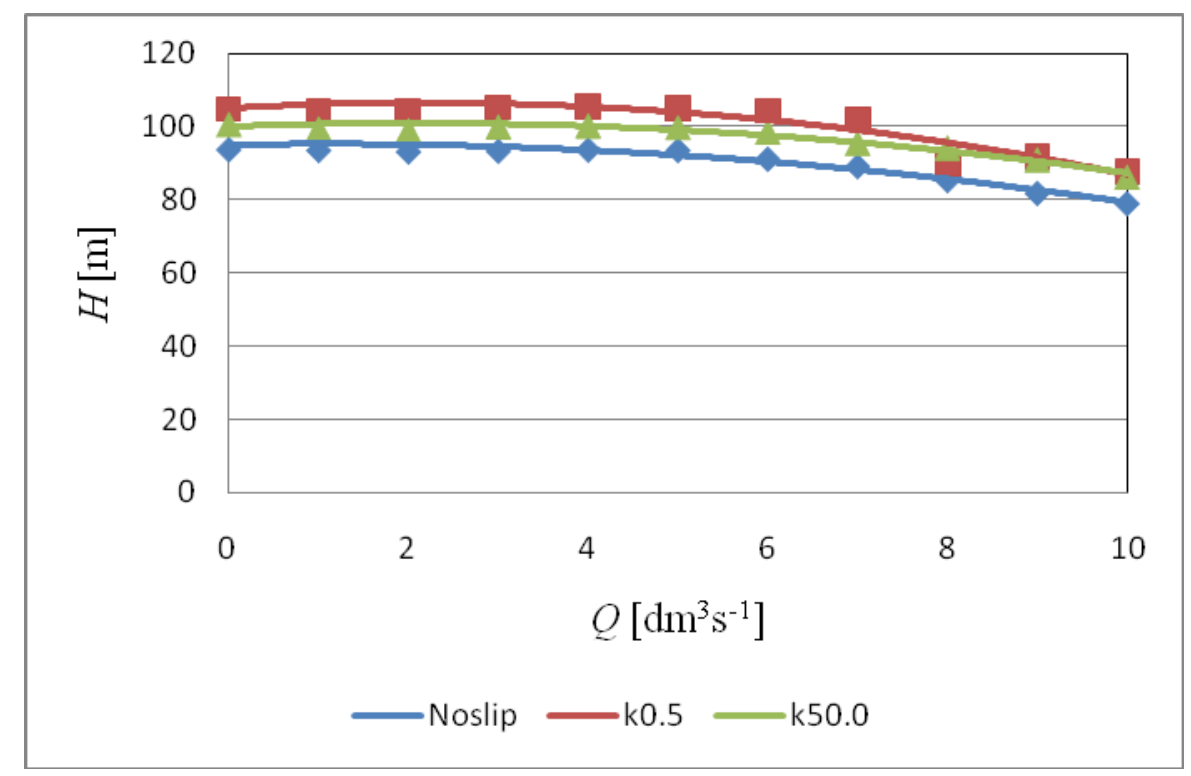

Fig. 9 Centrifugal pump characteristic

Conversely the adhesive coefficient reduction will significantly affect the hydraulic efficiency of centrifugal pump, by hydraulic losses lowering. See Fig.10

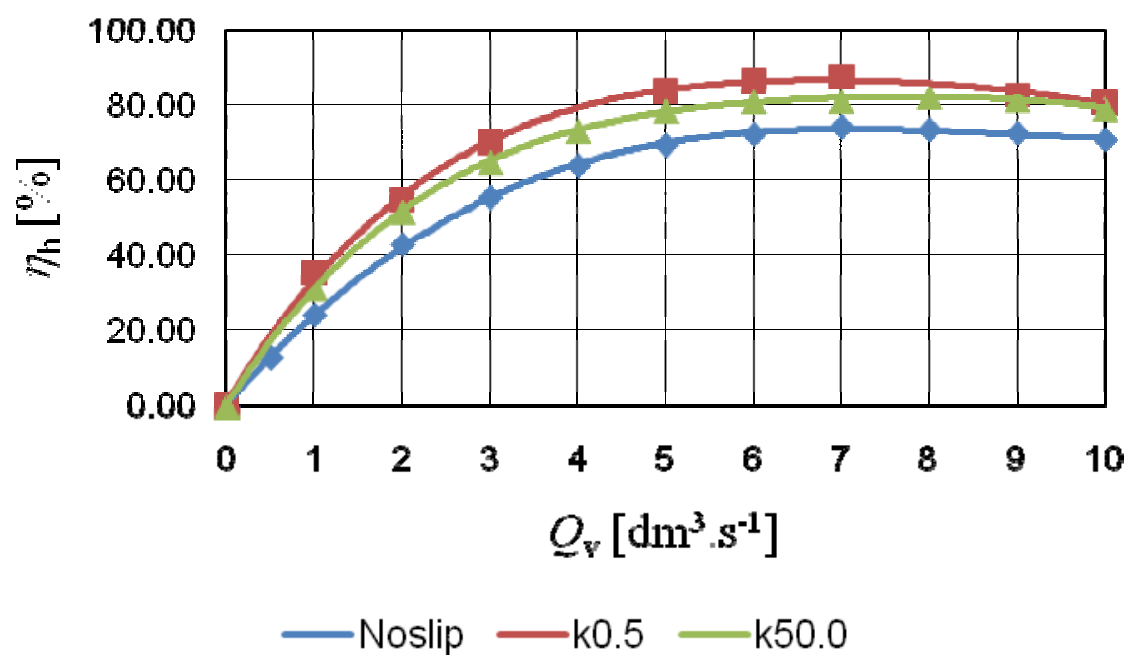

Fig. 10 Hydraulic efficiency characteristics of centrifugal pump

Another phenomenon that must be taken into account in the design of centrifugal pump is the dependence of radial force on the discharge. Fig. 11 shows the radial force increasing by the adhesive coefficient reducing. This phenomenon is mainly related to higher velocity rates of liquid close to the spiral body nose area. 


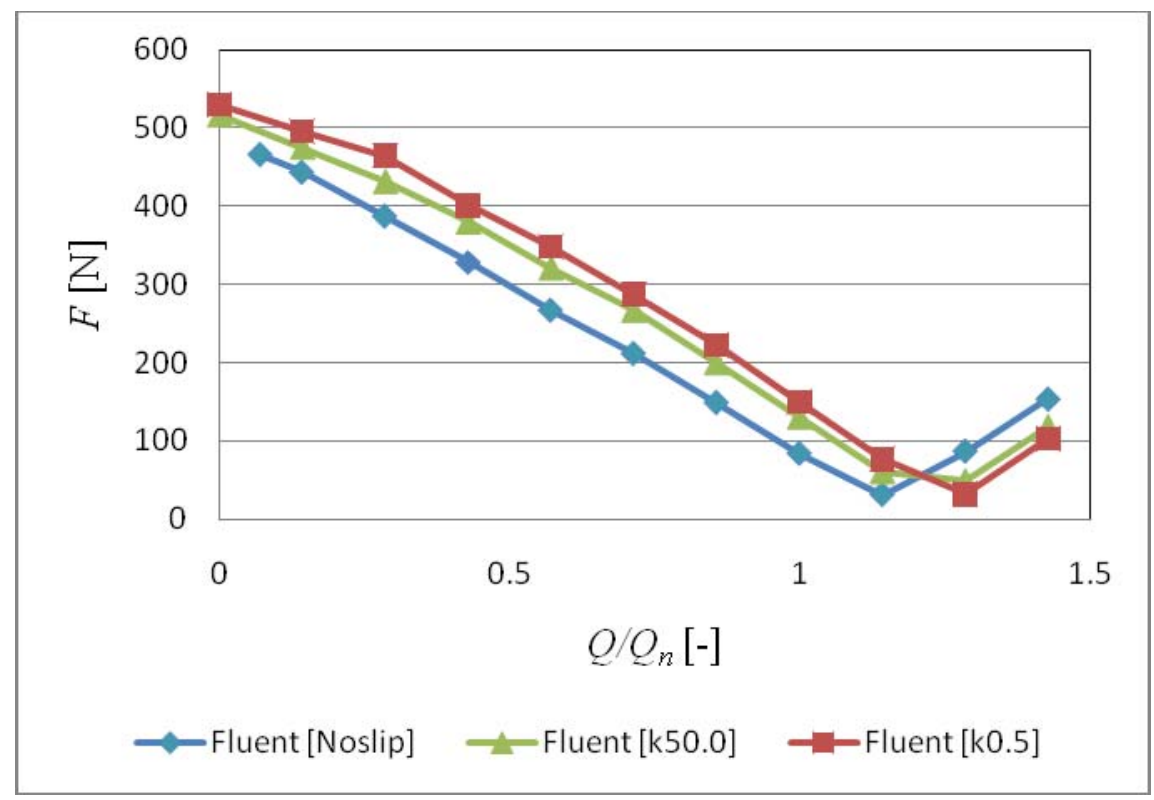

Fig. 11 Dependence of centrifugal pump radial force on discharge

In addition to the previous mathematical simulation, there was also carried out an experiment, this time for the pump with medium specific speed.

Fig.12 shows a centrifugal pump, where the interior parts were painted with a special low wettable material. Experiment results are shown in the next Figure 13, from which is obvious efficiency increase by $5 \%$ at the optimum operating point. Please note that this is a coating with a relatively high surface energy, so to increase the effectiveness may also help the surface roughness reduction.
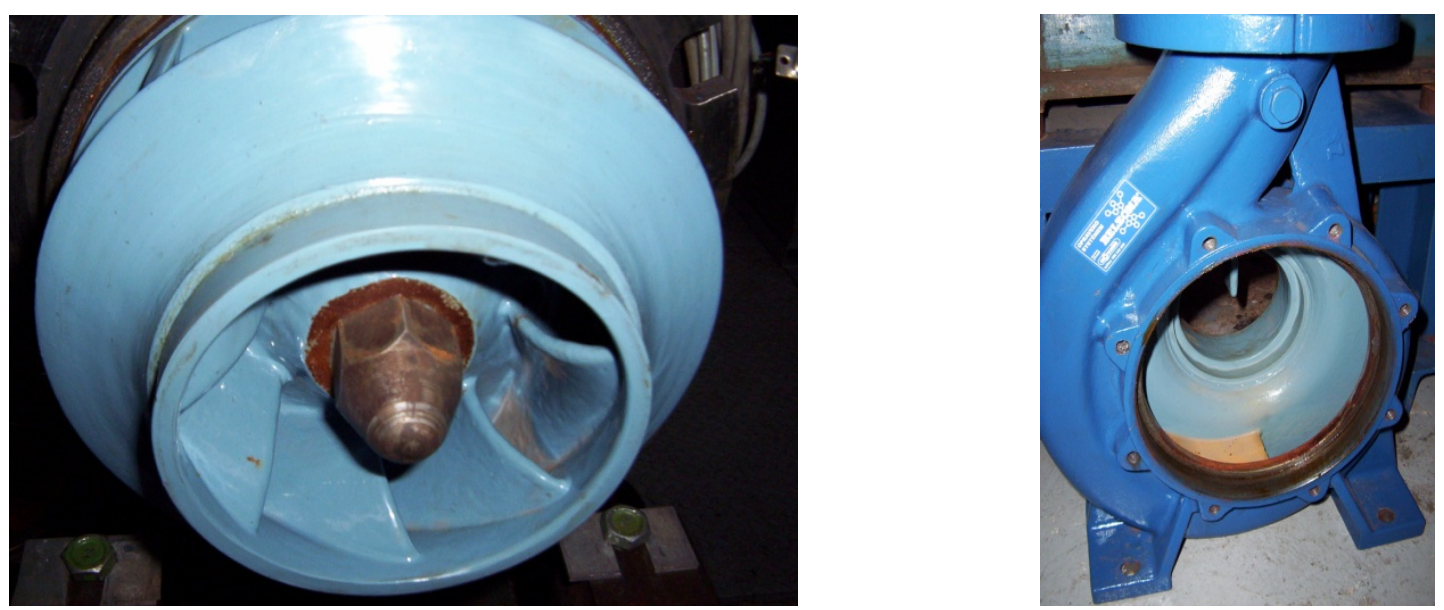

Fig. 12 Measured centrifugal pump with nonwettable interior 


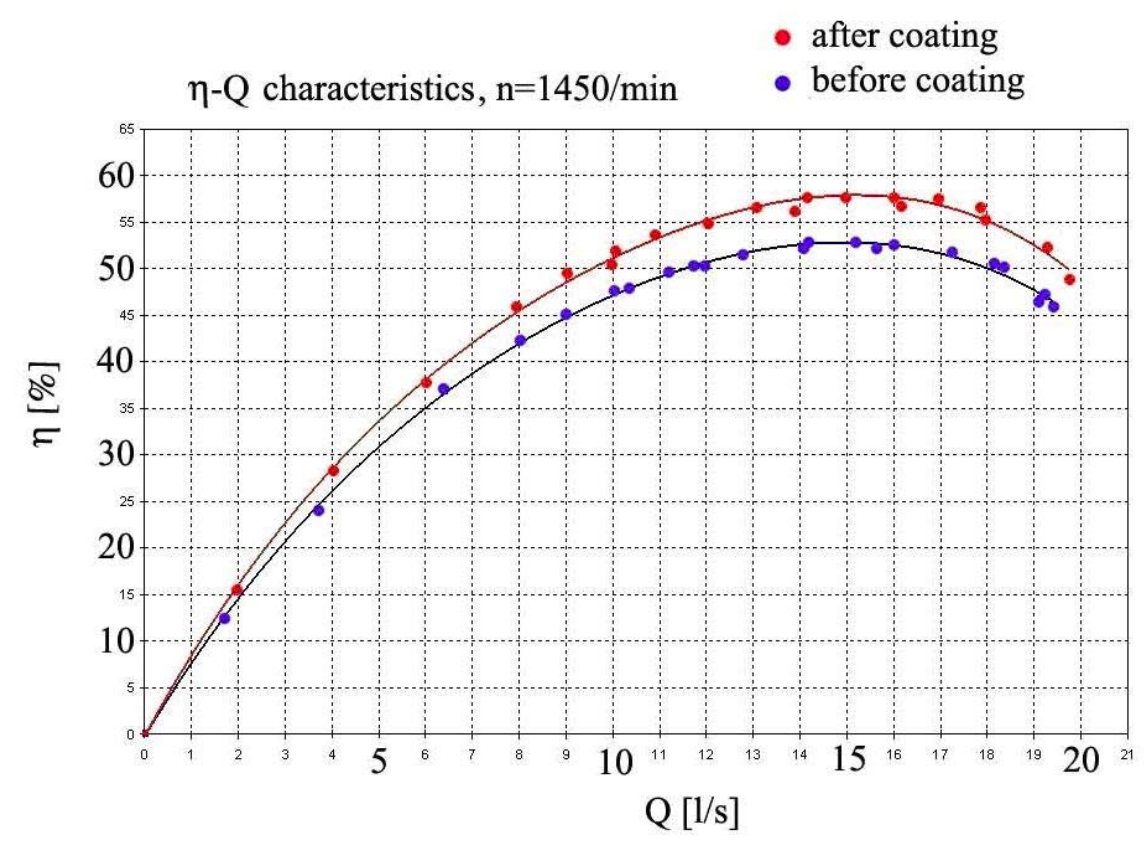

Fig. 13 Characteristics of centrifugal pump with medium specific speed

\section{Conclusion}

The past five years have seen a significant increase in nanotechnology applications to change the properties of surface layers. From the standpoint of fluid mechanics is appropriate to focus those technologies on such modification of surface layer in contact with liquid, which will repel the liquid. By this achieves the new quality of the fluid flow, which will be crucial in hydraulic losses reducing. This could result in significant energy savings in the hydraulic machines, components and equipment operation.

Non wettability of the liquid towards the surface brings new knowledge also in the theoretical field as fundamentally affect the idea of the turbulent flow creation and the current turbulence models based on wall functions loose the validity.

Flow in the liquid surface layer will affect the mechanism of cavitation. From this it is clear that conditions in the fluid boundary layer significantly affects the image of fluid flow at all and will have a fundamental impact on the construction of hydraulic components.

Adhesive coefficient effect is significant in another field of pump design computations where affects the centrifugal pump efficiency and the dependence of radial forces on discharge.

Remark: The adhesive coefficient dependence on the surface energy wasn't experimentally settled yet, because the proper methodic is still unknown.

\section{Acknowledgement}

Ministry of Education MSM 0021630518 and Czech Republic Grant Agency GA101/09/1715 are gratefully acknowledged for support of this work.

\section{Nomenclature}

$\begin{array}{ll}c & \text { Liquid velocity }\left[\mathrm{ms}^{-1}\right] \\ k & \text { Adhesive coefficient }\left[\mathrm{Pas} \mathrm{m}^{-2}\right] \\ \sigma & \text { Tension }\left[\mathrm{Nm}^{-2}\right] \\ p & \text { Pressure }\left[\mathrm{Nm}^{-2}\right] \\ \eta & \text { Viscosity }[\mathrm{Pas}]\end{array}$

$\begin{array}{ll}\Theta & \text { Contact angle }\left[{ }^{\circ}\right] \\ R, r & \text { Radiuses }[\mathrm{m}] \\ \rho & \text { Liquid density }\left[\mathrm{kg}^{-3}\right] \\ \Pi_{i j} & \text { Irreversible stress tensor }\end{array}$

\section{References}

[1] Young, T., Philos. Trans. R., Soc. London 95, 65 (1805)

[2] Van Oss, C.J., Good, R.J., Chaudhury, M.K., J. Colloid., Inter. Sci. 111 (1986) 378

[3] NAVIER, C.L.M.H., Mem. Acad. R. Sci. Inst. France 1 (1823) 414

[4] Pochylý, F., Fialová, S., Rinka, L., “Adhesive forces at a solid-liquid interface,” Zeszyty naukowe, ISSN 1897-2683, pp. 245252.

[5] Pochylý, F., Fialová, S., Habán, V., Rinka, L., “The wettability of the liquid-solid interface,” FIV 2008, ISBN 80-87012 12-7, pp. 47-52.

[6] Pochylý, F., Fialová, S., Habán, V., Rinka, L., "Praktičeskoje ispolzovanie smačivajemosti," PCVEXPO 08, ISBN 978-5-70383214-1. 
[7] Fialová, S., Pochylý, F., Rinka, L., “The adhesive force determination on the interface of phases,” ME 2008, ISBN 978-80227-2982-6.

[8] Fialová, S., Pochylý, F., Rinka, L., "The Boundary Conditions of the Adhesive Forces Effect on the Solid/Liquid Interface," Engineering mechanics 2009, ISBN 978-80-86246-35-2, pp. 241-246.

[9] POCHYLÝ, F., FIALOVÁ, S., KOZUBKOVÁ, M., ZAVADIL, L., “Assessment of cavitation creation depending on the surface wettability," In Proceedings of the 25th IAHR Symposium on Hydraulic Machinery and Systems, Timisoara, Romania, Editura Politehnica Timisoara, Romania, 2010, pp. 839-846, ISBN 978-606-554-136-8. 